\title{
The Effect of the Rear Cavity Wall Ingot Shape on the Evolution of the Liquid-Solid Interface During Melting for the Eutectic Pt-C
}

\author{
P. Castro, ${ }^{1,3}$ P. Bloembergen, ${ }^{2}$ A. Arroyo ${ }^{1}$
}

${ }^{1}$ University of Cantabria, Santander, Spain

${ }^{2}$ National Institute of Metrology, NIM, Beijing, 100013, China

${ }^{3}$ To whom correspondence should be addressed. E-mail: castropb@ unican.es 


\section{Abstract}

When characterizing high-temperature fixed points, the fraction of the melting time of the regular part of the plateau with respect to the total melting time, is critical. Maximizing the melting duration minimizes the uncertainty associated with the determination of the fixed-point temperature. One factor that affects this quality is the effect of the thermal bridging between the external and internal surfaces of the ingot enclosed by the cell. This paper presents the results of simulations for the eutectic Pt-C, investigating the effects of different ingot shapes on the duration of the melt plateau. It was found that the formation of a thermal bridge from the rear of the blackbody cavity towards the outer surface of the ingot was critical and that its formation could be delayed or suppressed through a proper choice of the ingot shape. The shapes considered included, firstly, the shape of the rear of the cavity, in contact with the ingot, either cone-shaped or dome-shaped, and secondly, the inside rear surface of the cell, in contact with the ingot, being a cone, a convex dome, or flat. The presence of impurities in the alloy was taken into consideration, and its influence in the evolution of the liquid-solid interface compared with that for the pure alloy. The effect of changing the thermal isolation of the cell, at its front side, was also considered. A dome-shaped surface for the rear of the cavity was found to be more favorable for the development of a regular melting front, in conjunction with the segregation of impurities during melting. At the rear of the cell, a flat surface ensures the back wall is the last to experience thermal bridging, resulting in more extended melting plateaus.

Keywords: High temperature fixed points; Liquid-solid interface; Shape effect 


\section{$1 \quad$ Introduction}

When characterizing high-temperature fixed points (HTFPs), the quality of the melting plateaus is a critical aspect to be considered. One factor that affects the quality is the effect of thermal bridging during melting between the external and internal surfaces of the ingot.

Thermal bridging and the influence of the rear cavity wall ingot shape for a Pt-C HTFP are studied in this paper. The analysis of the melting process was performed using a twodimensional (2D) axisymmetrical finite volume model, developed in ANSYS FLUENT and used in previous studies of HTFPs [1, 2]. The Pt-C\#4 cell involved was of hybrid construction [3, 4], shown in Fig. 1. It was modeled without any furnace to focus on the effect of the ingot shape for a pure alloy and also for an impure system. Critical factors as regards the backside shape of the ingot turned out to be (1) the shape of the ingot just behind the cavity: cone-shaped or domeshaped and (2) the shape of the backside of the ingot: cone, dome-shaped, or flat. Temperatures were derived from the areal average of the back wall of the cavity. During these operations the total mass of the ingot has been taken as invariant.

\section{$2 \quad$ Systems to be Modeled}

Six different cell configurations of the hybrid Pt-C cell were modeled combining the dome, cone, and flat shapes of the backside of the ingot summarized in Table 1.

The backside of the ingot was taken as adiabatic, the heat injection rate for melting was $Q=5000 \mathrm{~W} \cdot \mathrm{m}^{-2}$ around and along the outer cylindrical section of the ingot; it was preceded by a freeze with heat extraction rate $Q_{\mathrm{f}}=-3000 \mathrm{~W} \cdot \mathrm{m}^{-2}$. These rates correspond with offsets for melting and freezing of $+8.3 \mathrm{~K}$ and $-5 \mathrm{~K}$, respectively [5].

The effect of varying the thermal insulation of the ingot around its annular front side was also modeled, and two configurations were considered. Firstly, all the cell configurations were modeled with a heat loss of $Q=-2000 \mathrm{~W} \cdot \mathrm{m}^{-2}$ from the front. Secondly, to test the effect of heat loss from the front of the cell, just shape 1) in Table 1 was made with an adiabatic front wall.

Pure and impure systems were modeled. The impurity distribution was represented by an effective Scheil function [5]:

$$
T(t)-T_{\mathrm{E}}=m c F(t)^{k-1}
$$


where $T$ and $t$ represent the temperature and time, respectively, $F$ is the liquid fraction, $T_{\mathrm{E}}=2012.229 \mathrm{~K}$ is the eutectic temperature of the pure system, $c=94 \times 10^{-6} \mathrm{~mol}^{-1}$ is the impurity concentration, $m=-0.915 \times 10^{-6} \mathrm{mK} \cdot \mathrm{mol}$ is the associated liquidus slope, and $k=0.316$ is the distribution coefficient. These parameters were chosen for modeling the effect of impurities and were taken from real data [5].

\section{$3 \quad$ Results}

Figures $2 \mathrm{a}$ and $\mathrm{b}$ show the melting curves of the impure and pure systems, respectively for the six ingot configurations considered as a function of time. The temperature along the vertical axis represents the temperature of the back wall of the cavity, $T_{\text {backwall }}(t)$. Figures $3 \mathrm{a}$ and $\mathrm{b}$ show the same curves as a function of the liquid fraction $F=\left(t-t_{\mathrm{sol}}\right) /\left(t_{\mathrm{liq}}-t_{\mathrm{sol}}\right)$, where $t_{\mathrm{sol}}$ and $t_{\text {liq }}$ are the times corresponding to the solidus and liquidus points, characterized by the maximum in slope of the measured curve, just before melting, and just after completion of melting, respectively. Values of the characteristic times and liquid fraction in the melting curve for impure and pure systems are presented in Table $2 \mathrm{a}$ and $\mathrm{b}$.

The contours delimiting the liquid phase for the impure system during melting at $t_{\text {bridge, }}$ at which thermal bridging is initiated, are given in Fig. 4 for all the configurations 1) to 6).

The prime parameter to judge the effect of varying the backside of the shape of the ingot is the liquid fraction $F\left(t_{\text {bridge }}\right)=\left(t_{\text {bridge }}-t_{\text {sol }}\right) /\left(t_{\text {liq }}-t_{\text {sol }}\right)$, where $t_{\text {bridge }}$ is the abrupt upturn in the melting curve, where thermal bridging is initiated, followed by the post-melting part of the curves, see Figs. 2 and 3. A second effect interfering with thermal bridging and to be taken into account is inside melting, which is effectively an impurity effect [5]. It is interesting to note that the upturn is closer to $F=1$ for the flat-backside configurations.

A comparison between the pure and impure systems with the liquid fraction for configurations 1) and 6) is shown in Fig. 5, where lower melting temperatures and more abrupt upturns are observed for the impure system.

The effect of thermal isolation on the right-hand side of the cell turned out to be small for the calculated values: $Q=-2000 \mathrm{~W} \cdot \mathrm{m}^{-2}$ and adiabatic conditions. Both melting plateaus for the impure system plotted versus time and liquid fraction are shown in Figs. 6 and 7, respectively. 


\section{Discussion}

It is shown in Fig. 4 that dome-shaped cavities develop a more homogeneous melting front around the cavity, reducing the possibility of thermal bridging and overheated cavity walls. On the other hand, flat backside ingots have the effect on the region behind the back wall of the cavity to be the last to melt, because of the extra material concentrated near to the back edge of the ingot, resulting in more extended melting plateaus as can be seen in Figs. 2 and 3.

The abrupt upturn is associated with thermal bridging between the inside of the ingot near the back wall of the cavity and the outside of the ingot, allowing heat to flow from the surroundings, at a higher temperature, towards the ingot. This is aggravated by the presence of impurities as can be seen in Fig. 5, causing an inside melting front to develop before the end of melting, since the bridging is triggered already when the inside front touches the outside front, see Fig. 4.

It should be stressed here that -in contrast with the impure system- the pure system shows outside melting only, by which the upturn associated with thermal bridging for the pure system is more gradual than that of its impure counterpart (Figs. 3a, 3b, 5). On the other hand, the more gradual evolution of the melting process towards the melting plateau in the latter case is just an impurity effect, characteristic for Scheil freezing preceding melting. It is interesting to note that the plateau in terms of liquid fraction is extended further towards $F=1$ for the impure system than for the corresponding pure system, see Figs $3 a, 3 b$, and 5 and Tables $2 a$ and $b$.

The usual flat backside configuration turned out to be a good solution for keeping the melting plateau stabilized almost over the whole liquid fraction range, Fig. 3. The dome wall behind the cavity restricts the effect of thermal bridging, preventing an overheated cavity back wall in an early stage of the melting process, so a flat-dome configuration -number 2) in this study- is recommended -as a compromise- to include both these advantages. Increasing the amount of ingot material at the backside of the ingot -and thus increasing its total mass- may further help in optimizing the system. Finally one might suspect that during use at high temperature, the back wall of the cavity in contact with the ingot would erode in time into a more rounded shape by which a more optimum configuration would be obtained. 


\section{$5 \quad$ Conclusions}

Modeling has shown that optimum performance for HTFPs is better obtained by having a flat back wall of the ingot and a dome shape behind the cavity in contact with the metal ingot. Impurities can induce inside melting confounding the effect of thermal bridging. 


\section{References}

1. P. Castro, G. Machin, M.A. Villamañan, D. Lowe, Int. J. Thermophys. 32, 1773 (2011)

2. P. Castro, P. Bloembergen, Y. Yamada, M.A. Villamañan, G. Machin, Acta Metrol. Sinica 29, 253 (2008)

3. W. Dong, P. Bloembergen, T. Wang, Y.Y. Duan, Int. J. Thermophys. 32, 2680 (2011)

4. W. Dong, T. Wang, P. Bloembergen, Y.Y. Duan, submitted to Metrologia

5. P. Castro, P. Bloembergen, W. Dong, "The Effect of Impurities on the Evolution of the Melting Front, Analysed in a Two-dimensional Representation for the Eutectic Pt-C," submitted to Int. J. Thermophys. 
Table 1 List of cell configurations

\begin{tabular}{ccc}
\hline No. & Backside of the ingot & $\begin{array}{c}\text { Ingot behind the } \\
\text { cavity }\end{array}$ \\
\hline 1) & Flat & Cone \\
2) & Flat & Dome \\
3) & Cone & Cone \\
4) & Cone & Dome \\
5) & Dome & Cone \\
6) & Dome & Dome \\
\hline
\end{tabular}


Table 2 (a) Characteristic times and liquid fraction $F$ in the melting curve for impure system, shown in Figs. 2a and 3a

\begin{tabular}{ccccc}
\hline $\begin{array}{c}\text { Cell configuration } \\
\text { No. }\end{array}$ & $\boldsymbol{t}_{\text {sol }}(\mathbf{s})$ & $\boldsymbol{t}_{\text {bridge }}(\mathbf{s})$ & $\boldsymbol{t}_{\text {liq }}(\mathbf{s})$ & $\begin{array}{c}\text { Upturn } \\
\left.\boldsymbol{t}_{\text {bridge }}\right)\end{array}$ \\
\hline $\mathbf{1})$ & & & 2180 & 0.95 \\
$\mathbf{2}$ & 1450 & 2145 & 2175 & 0.90 \\
$\mathbf{3})$ & 1440 & 2100 & 2155 & 0.82 \\
$\mathbf{4})$ & 1370 & 2010 & 2185 & 0.79 \\
$\mathbf{5 )}$ & 1390 & 2015 & 2140 & 0.83 \\
$\mathbf{6}$ & 1375 & 2010 & 2135 & 0.82 \\
\hline
\end{tabular}

(b) Characteristic times and liquid fraction $F$ in the melting curve for pure system, shown in Figs. $2 b$ and $3 b$

\begin{tabular}{ccccc}
\hline $\begin{array}{c}\text { Cell configuration } \\
\text { No. }\end{array}$ & $\boldsymbol{t}_{\text {sol }}(\mathbf{s})$ & $\boldsymbol{t}_{\text {bridge }}(\mathbf{s})$ & $\boldsymbol{t}_{\text {liq }}(\mathbf{s})$ & Upturn \\
\hline $\mathbf{1})$ & & & $\boldsymbol{F}\left(\boldsymbol{t}_{\text {bridge }}\right)$ \\
\hline $\mathbf{2})$ & 1225 & 1480 & 1530 & 0.84 \\
$\mathbf{3})$ & 1215 & 1470 & 1515 & 0.85 \\
$\mathbf{4})$ & 1185 & 1428 & 1512 & 0.74 \\
$\mathbf{5 )}$ & 1190 & 1430 & 1513 & 0.74 \\
$\mathbf{6}$ & 1195 & 1432 & 1513 & 0.74 \\
\hline
\end{tabular}




\section{Figure Captions}

Fig. 1 Schematic diagram of the Pt-C\#4 cell

Fig. 2 (a) Melting plateaus of the impure system for the six configurations versus time and (b) melting plateaus of the pure system for the six configurations versus time

Fig. 3 (a) Melting plateaus of the impure system for the six configurations versus the liquid fraction $\mathrm{F}$ and (b) melting plateaus of the pure system for the six configurations versus the liquid fraction $F$

Fig. 4 Contours delimiting the liquid phase (blue solid, red liquid) during melting at $t=t_{\text {bridge }}$ for the six configurations with the impure system, as specified in Table 1

Fig. 5 Melting plateaus of the pure and impure systems versus the liquid fraction $F$ for configurations 1) and 6)

Fig. 6 Melting plateaus for two different insulations at the front side of the ingot versus time for cell 1

Fig. 7 Melting plateaus for two different insulations at the front side of the ingot versus the liquid fraction $F$ for cell 1 


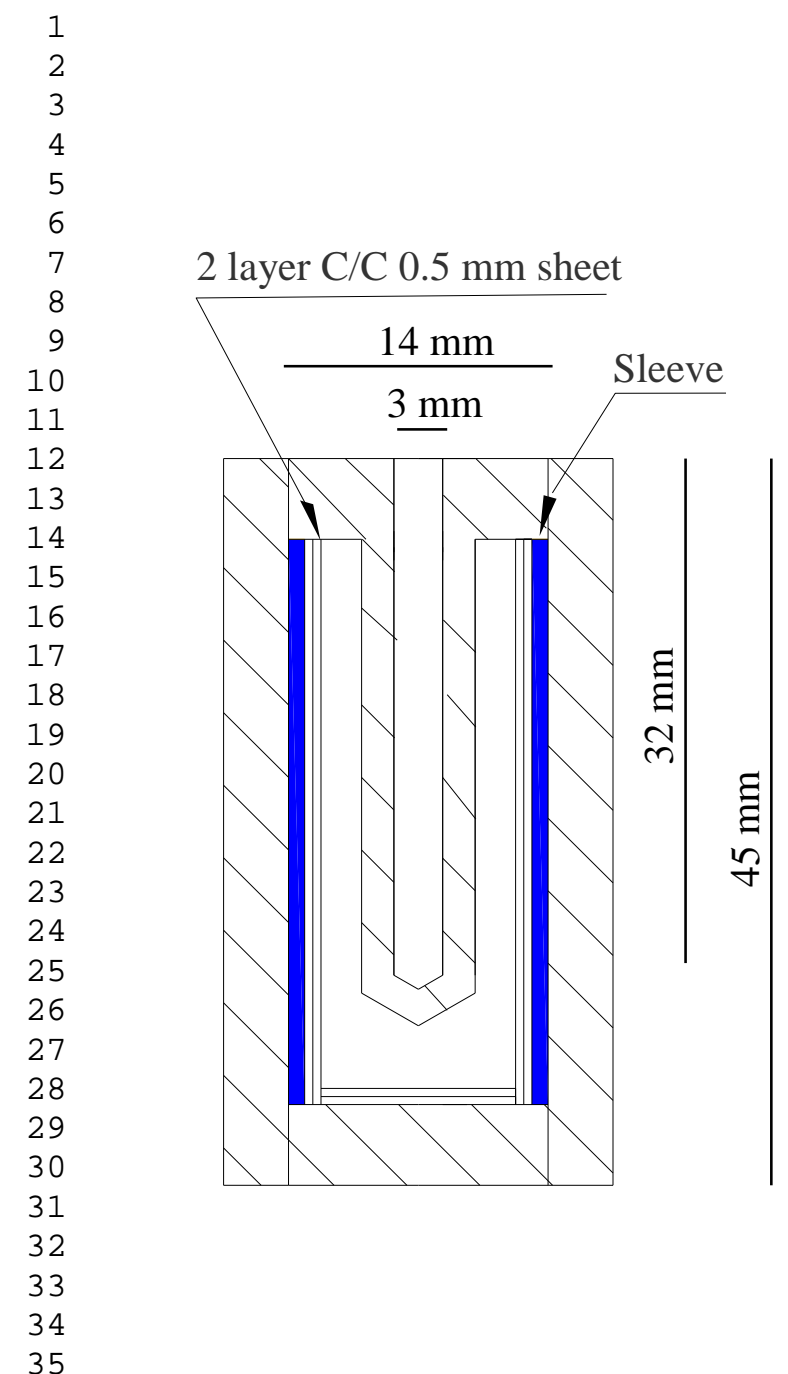

Fig. 1

36

37

38

39

40

41

42

43

44

45

46

47

48

49

50

51

52

53

54

55

56

57

58

59

60

61

62

63

64

65 


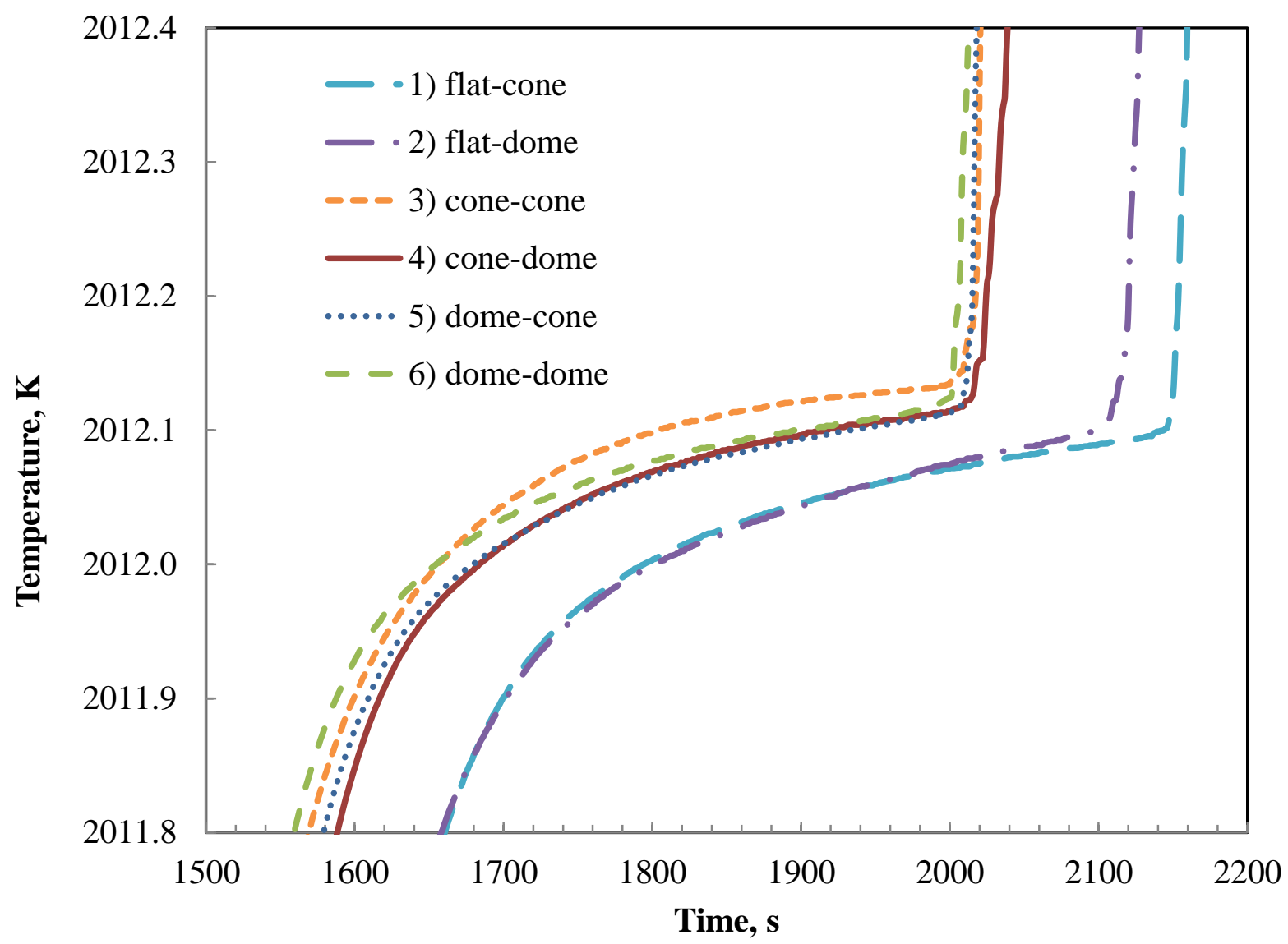

(a)

Fig. 2a 


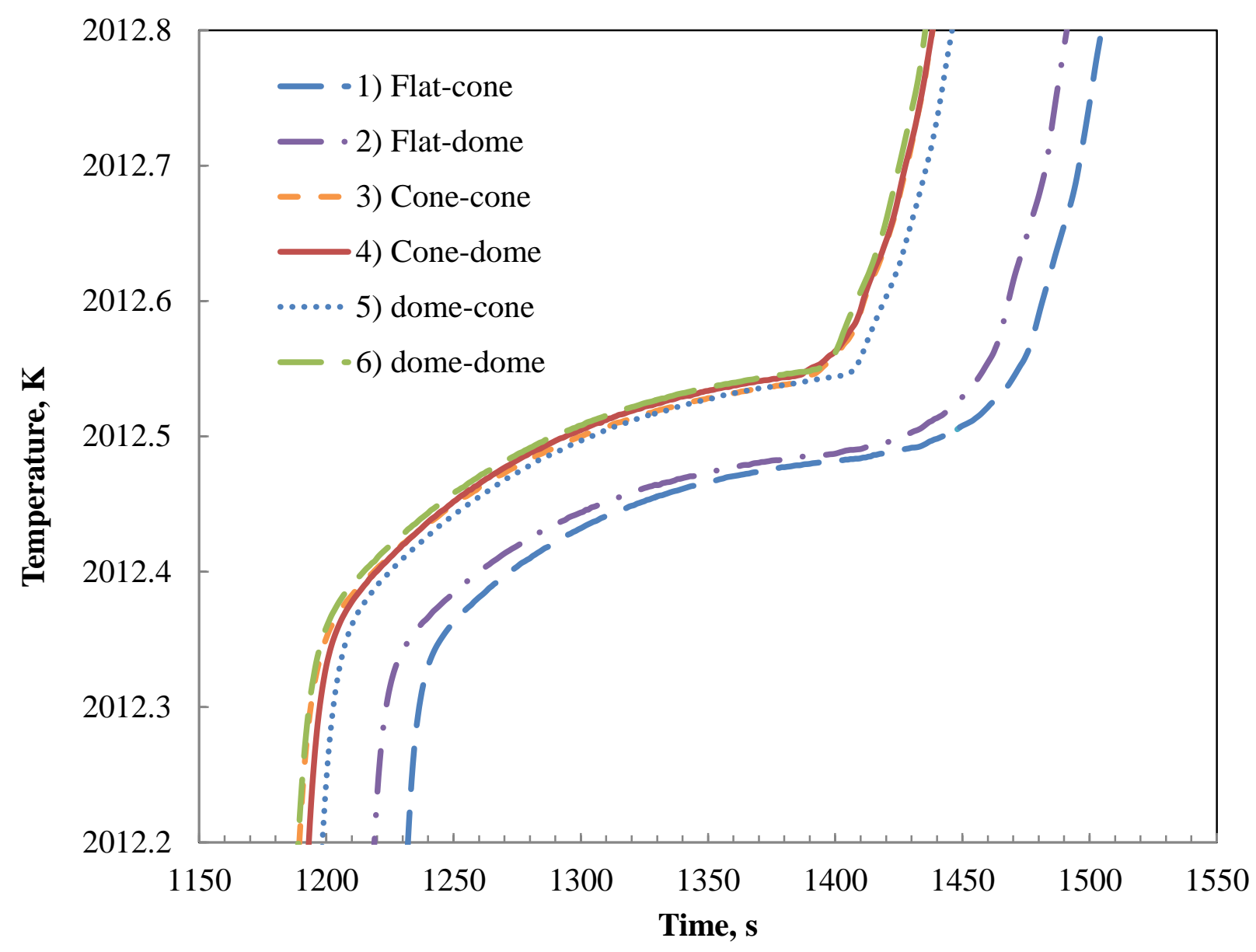

(b)

Fig. 2b 


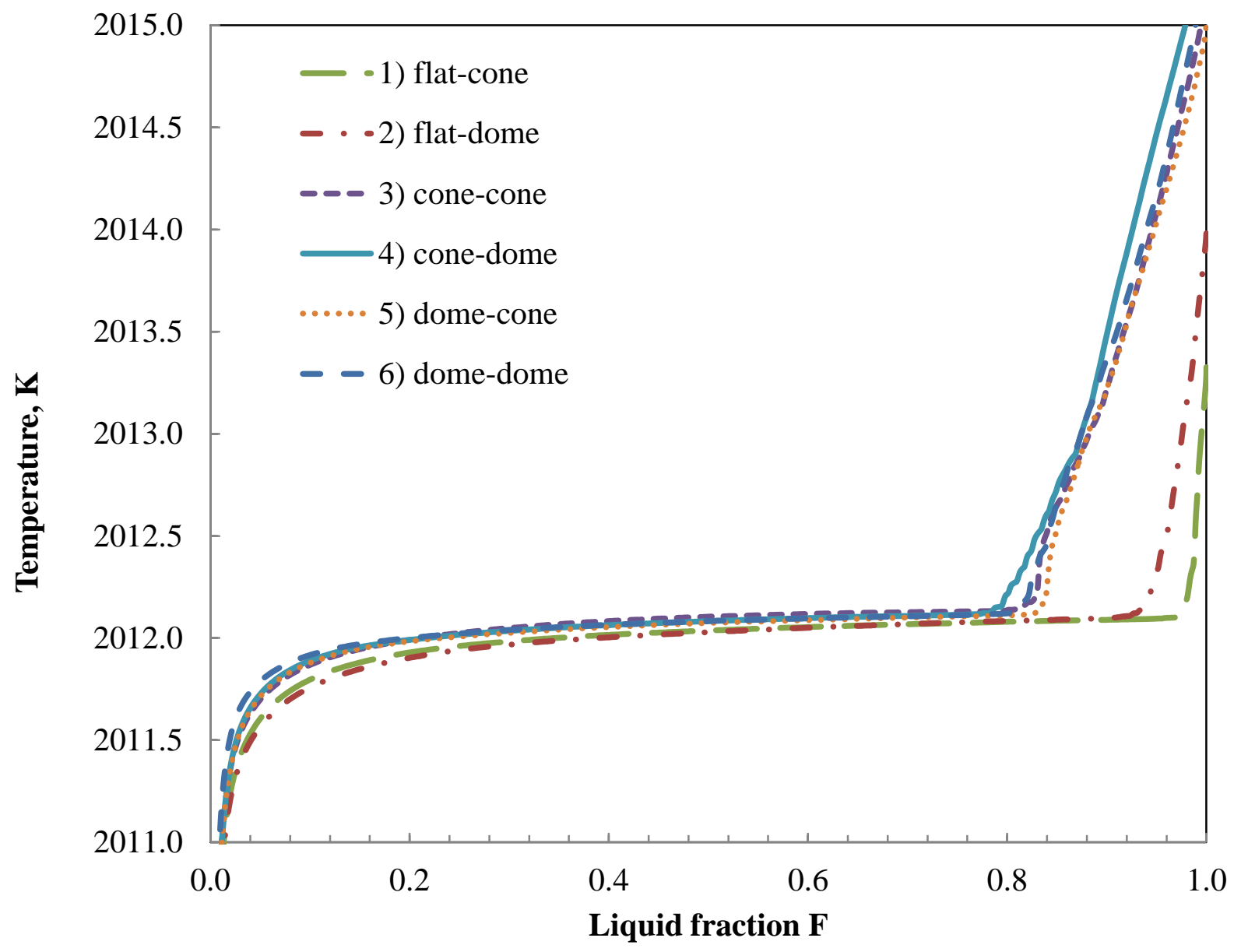

(a)

Fig. 3a 


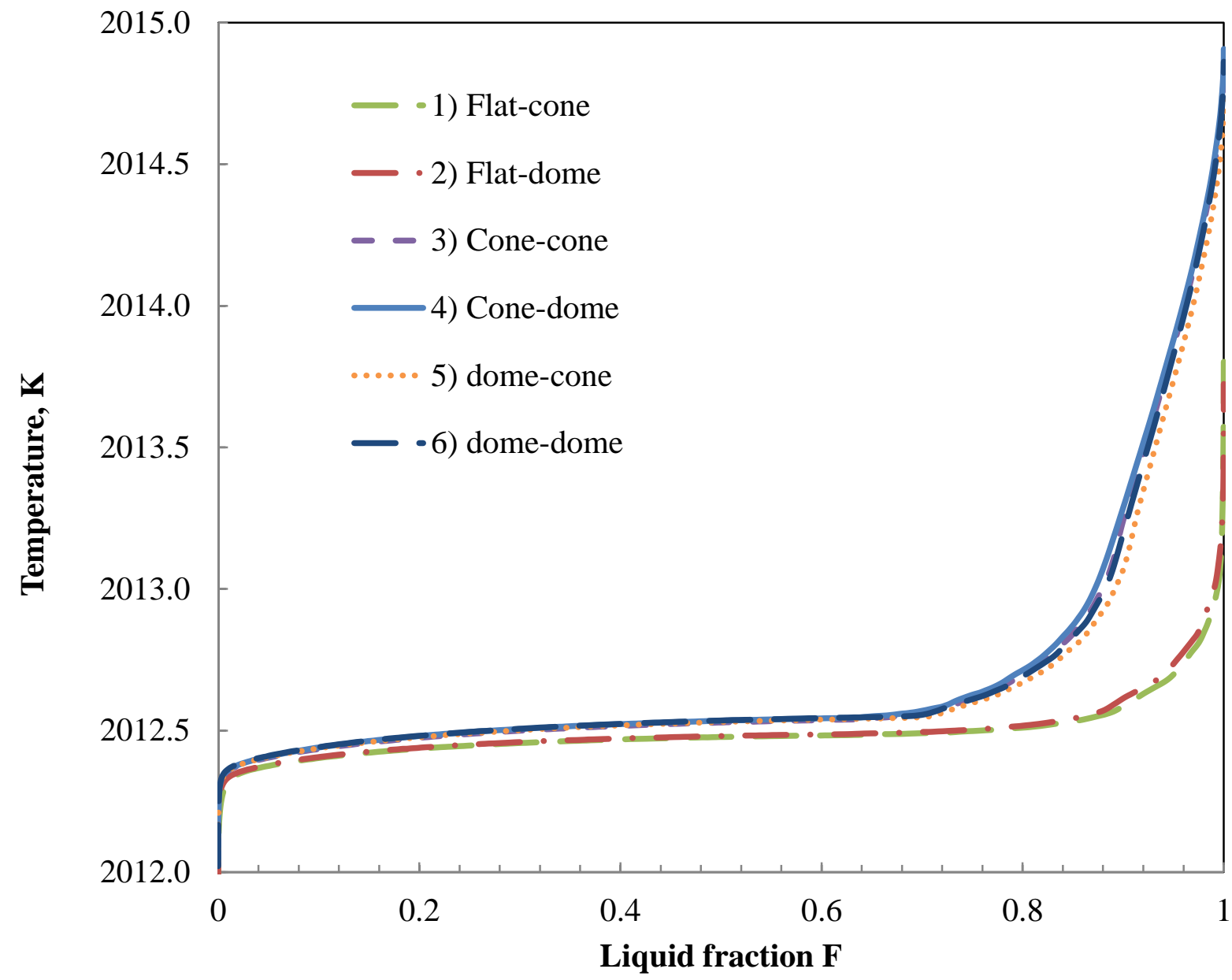

(b)

Fig. $3 b$ 
1)

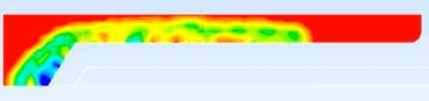

3)

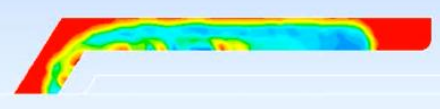

5)

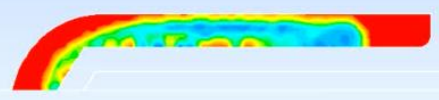

2)

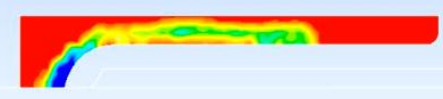

4)

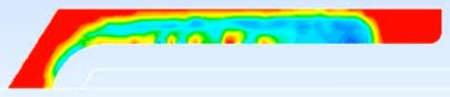

6)

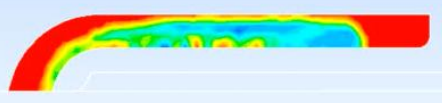

Fig. 4

29

30

31

32

33

34

35

36

37

38

39

40

41

42

43

44

45

46

47

48

49

50

51

52

53

54

55

56

57

58

59

60

61

62

63

64

65 


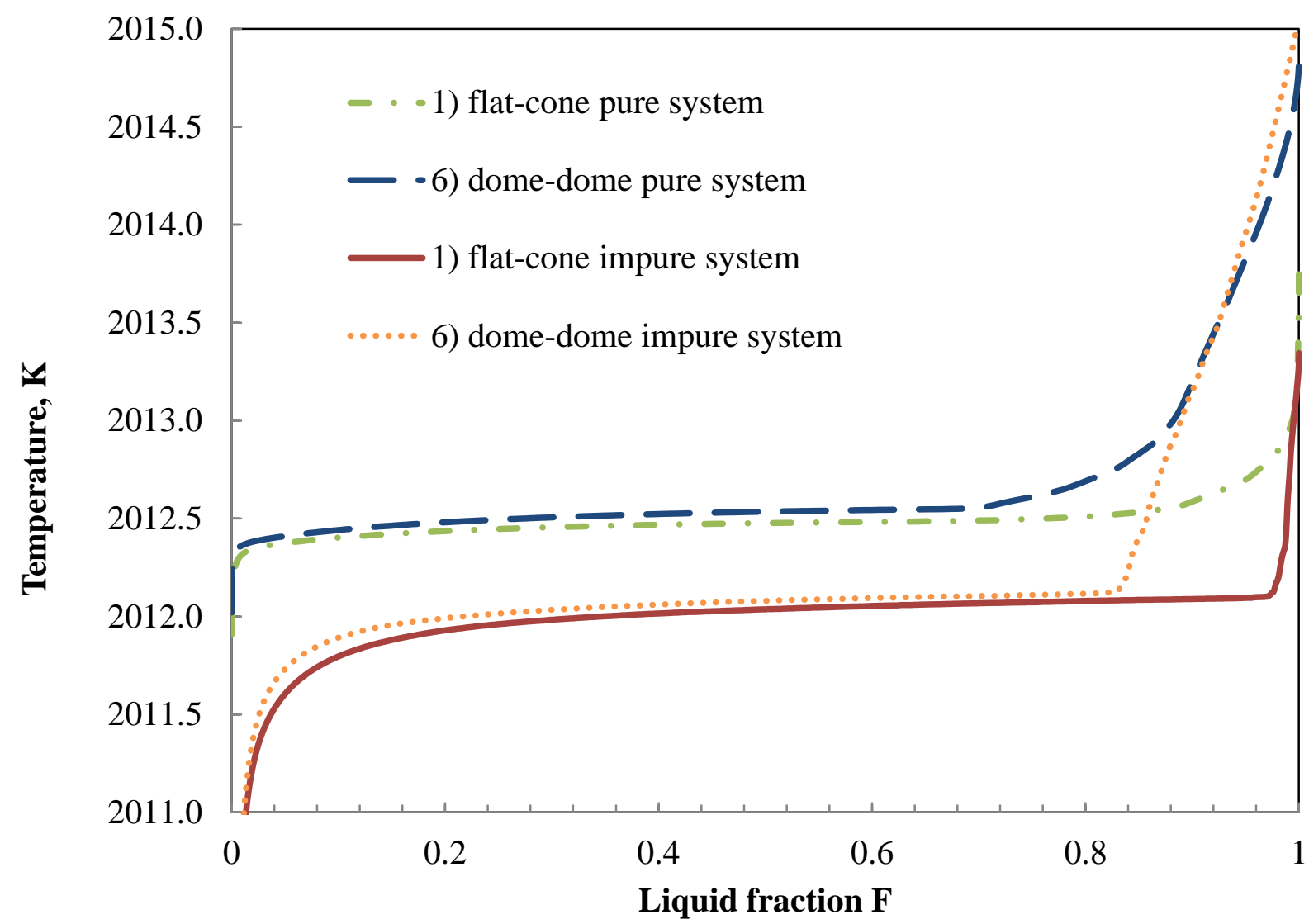

Fig. 5 


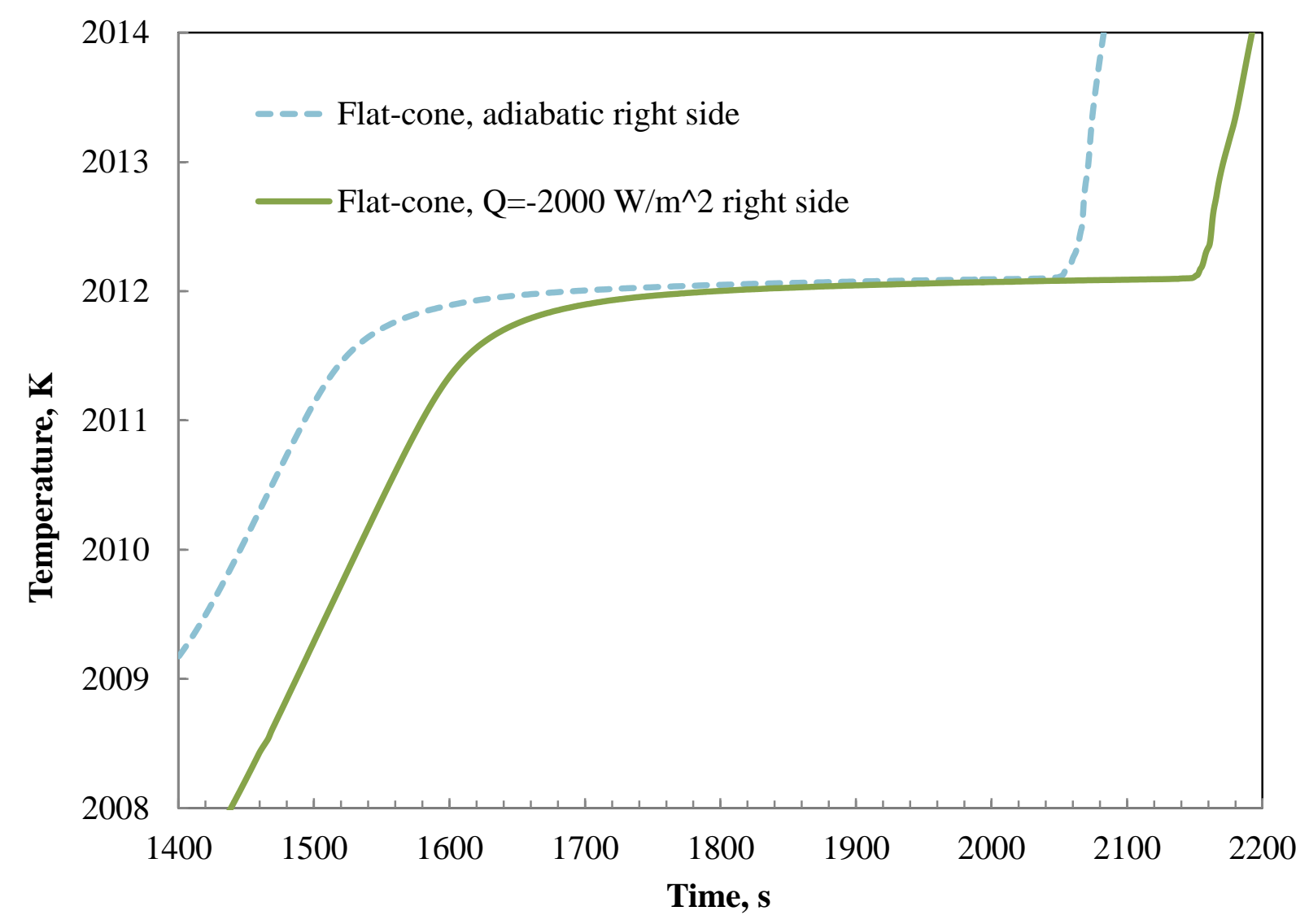

Fig. 6 


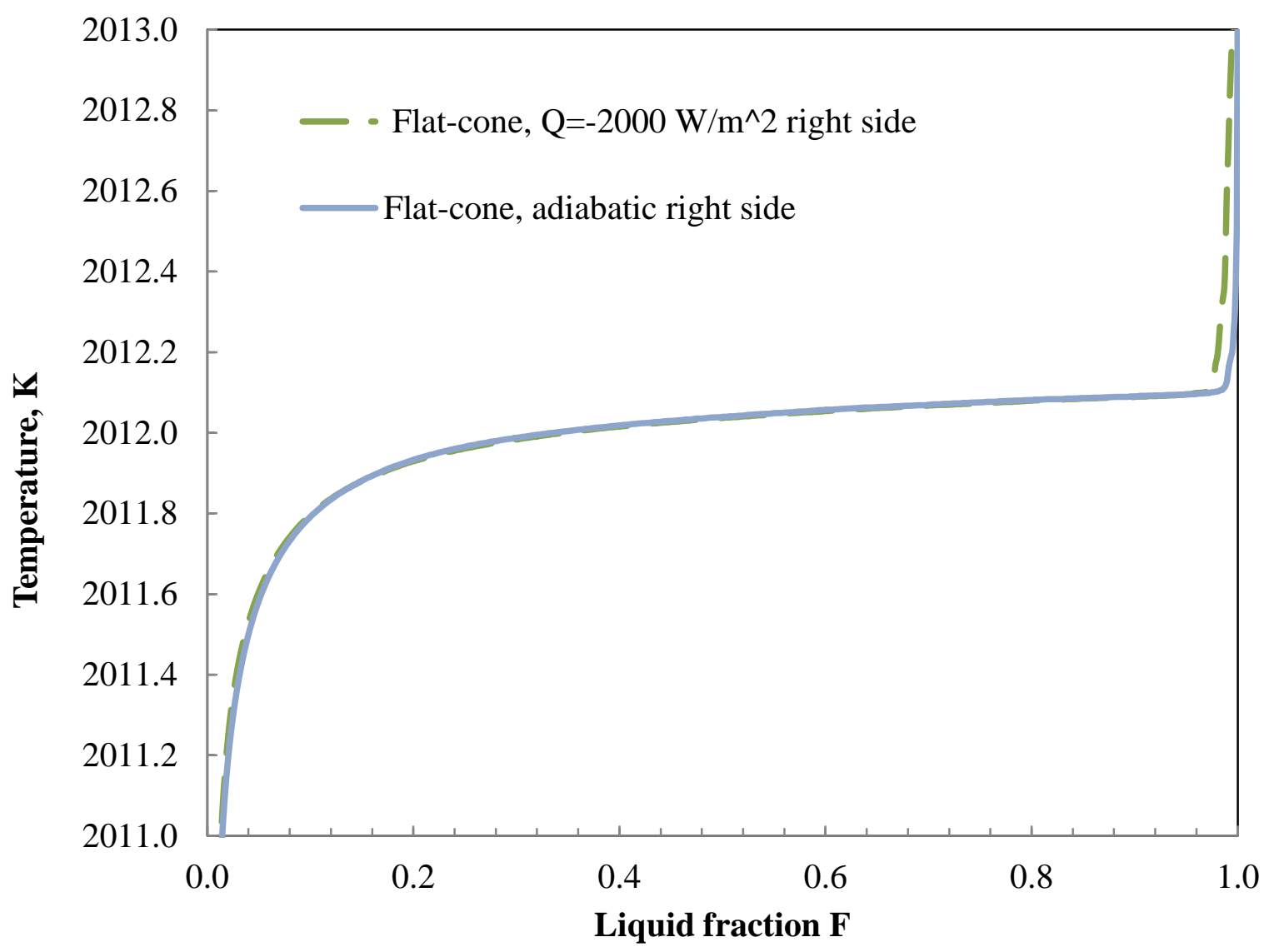

Fig. 7 